\title{
The locus of context effects on product proximity judgments
}

\author{
Lauren Goldberg Block \\ Stern School of Business, New York University, New York, NY 10012-1126, USA \\ Michael D. Johnson \\ Department of Marketing, School of Business Administration, The University of Michigan, 701 Tappan \\ Street, Ann Arbor, M148109-1234, USA
}

\begin{abstract}
Tversky's (Tversky, A., 1977. Features of similarity. Psychological Review 84, 327-352) contrast model of similarity is used to examine the locus of two contextual effects on product judgments. More familiar stimuli may be perceived as more similar as well as more dissimilar than less familiar stimuli. Priming subjects' product knowledge should, meanwhile, increase the similarity of brands but decrease the similarity of categories. We argue that both of these context effects should be more prominent among the moderately proximal stimuli in any given set. Two studies, one examining the similarity/dissimilarity effect and one examining priming effects, support this conclusion. The observed priming effects were also stronger for brands than for categories. Overall these context effects appear more localized than previously assumed.
\end{abstract}

\section{Introduction}

Proximity measures are used in a wide variety of consumer and market research domains.

Traditionally they have served as input to multidimensional scaling and clustering techniques in order to examine product perceptions and market structure (Cooper, 1983; Shocker and Srinivasan, 1979). More recently, proximity measures have been used in research on choice models (Glazer et al., 1991; Tversky 
and Sattath, 1979), categories and categorization (Barsalou, 1983, 1985; Loken and Ward, 1990; Rosch, 1975, 1977), and brand equity (Aaker and Keller, 1990; Keller, 1991). Understanding how consumers judge proximity remains an important market research question.

A prominent psychological model of similarity, the contrast model (Tversky, 1977), has been proposed to explain how individuals make proximity judgments. The contrast model views proximity judgments as a comparison or contrast of common and distinctive features. A major advantage of the contrast model is its ability to explain and predict the influence of context and task effects on proximity judgments. This has important implications as there are many situations, such as comparative advertising, in which one may exert control over the context of a comparison. However, it is unclear just how pervasive these context effects are.

This paper examines the locus of context effects on product proximity judgments. We focus on two types of effects, a similarity/dissimilarity effect and a priming effect. Tversky (1977; Tversky and Gati, 1978) demonstrates that more familiar stimuli may be judged as both more similar and more dissimilar than less familiar stimuli. Priming attribute associations should, meanwhile, increase the perceived proximity of similar brands but decrease the perceived proximity of dissimilar categories. The contrast model framework is used to argue that these context effects should vary as a function of both the relative proximity of the products in a set and their categorical level. Two studies, one examining the similarity/dissimilarity effect and one examining priming effects, indicate that the effects are concentrated at a moderate level of relative proximity. Priming is also shown to have a greater effect on the proximity of brands than on the proximity of categories. The results have implications for those concerned about the degree to which context affects product judgments, how we operationalize proximity and proximity related constructs in marketing research, and similarity scaling.

\section{Study one: Similarity versus dissimilarity}


Traditional geometric models of proximity presume that similarities are a direct reflection of dissimilarities. Yet Tversky and others (Gati and Tversky, 1984; Medin et al., 1990; Tversky, 1977; Tversky and Gati, 1978, 1982) demonstrate systematic violations of this relationship. A classic example involves sets of countries where each set contains a prominent or familiar pair of countries and an unfamiliar pair (e.g., East and West Germany versus Ceylon and Nepal). Tversky and Gati (1978) asked one group of subjects to select which of the two pairs was more similar while another group selected which of the two pairs was more different. On average, the more familiar pairs were selected more frequently in both conditions. Johnson (1981) reports a similar violation of consistency between similarities and dissimilarities in a consumer products context, particularly for soft drinks.

Tversky's (1977) contrast model helps explain this and other contextual effects. He argues that, when faced with a similarity or dissimilarity judgment, people extract from memory a limited list of relevant features to perform the task. The contrast model views proximity judgments as a comparison or contrast of these extracted features. Common features add to similarity while distinctive features detract. Likewise, distinctive features add to dissimilarity while common features detract. Stated formally as a linear model, the similarity between any two stimuli a and $b, s(a, b)$, associated with feature sets $A$ and $B$, respectively, is:

$s(a, b)=\theta f(A \cap B)-\alpha f(A-B)-\beta f(B-A)$

where $(A \cap B)$ are those features common to a and $\mathrm{b},(A-B)$ are those features distinctive to a, $(B-A)$ are those features distinctive to $\mathrm{b}, f$ is a function describing the measure or relative contribution of the individual features toward the proximity judgment, and $\theta, \alpha$, and $\beta$ are weights on 
the different common and distinctive feature sets. Analogously, the dissimilarity between any two stimuli a and $b, d(a, b)$, may be described as:

$d(a, b)=\alpha f(A-B)-\beta f(B-A)-\theta f(A \cap B)$.

A key feature of the contrast model is that the relative weights placed on the feature sets $(\theta, \alpha$, and $\beta$ ) are a function of the particular judgment task or context. For similarity judgments, it is natural to place relatively more weight on common features $(\theta>\alpha+\beta)$. When judging dissimilarity, distinctive features should be relatively more important $(\alpha+\beta>\theta)$. Thus, a pair of stimuli that has a large measure of both common and distinctive features may be judged as relatively similar in a similarity context as well as relatively dissimilar in a dissimilarity context.

The implication is that the proximity measure one obtains may be highly dependent on the nature of the judgment task. Medin et al. (1990) go as far as to argue that similarities and differences should not be viewed as interchangeable. Malhotra (1990) also argues that these two judgments are quite different in market research applications. However, the contrast model only predicts such a context effect when there is significant variance in the feature measures of the stimuli, or the function in Eqs. (1) and (2), which reflects the salience or prominence of the various features associated to the stimuli. The effect may be quite localized such that similarities generally mirror dissimilarities. Hosman and Kuennapas (1972; reported in Tversky, 1977), for example, found a correlation of -0.98 between independent judgments of the similarity and dissimilarity of lower case letters. Even Tversky and Gati (1978) found a similarity/dissimilarity correlation of -0.98 across all twenty-one pairs of countries in their study.

\subsection{The locus of the similarity/dissimilarity effect}


The important question is to what degree this context effect pervades consumer judgments. The contrast model predicts consistency between similarities and dissimilarities whenever stimuli have equivalent feature measures (Tversky, 1977). Put differently, when the common and distinctive features among a set of stimuli are equally prominent or salient, similarity and dissimilarity judgments should be highly related if not interchangeable. Only when certain stimuli in a set have more prominent or salient common as well as distinctive features than other stimuli should they be perceived and judged as more similar and dissimilar.

We identify a similarity/dissimilarity context effect by the degree to which the average similarity plus average dissimilarity (i.e., $[s(a, b)+d(a, b)]$ ) rating for different product pairs increases with the familiarity of the pairs. This suggests the following base hypothesis.

$\mathrm{H} 1$ : The perceived similarity plus dissimilarity of product pairs, $[s(a, b)+d(a, b)]$, increases with their relative familiarity.

Note that the hypothesis predicts that similarity plus dissimilarity will increase rather than predicting that both similarity and dissimilarity will increase. The similarity plus dissimilarity measure is consistent with Tversky's (1977) predictions. It also takes into account the likelihood that familiarity has another systematic effect on product proximity judgments. Product categorization is generally viewed as a process of grouping and distinguishing (Howard, 1977). As familiarity increases, differences among the members of a category become more salient and, over time, subcategories develop (Rosch, 1975, 1977). As consumers become more familiar with a category of products, we expect that the perceived similarity (dissimilarity) among the products should decrease (increase). It is unlikely, therefore, that Tversky's 
context effect will result in net increases in both similarity and dissimilarity with familiarity. Similarity plus dissimilarity should be a more sensitive measure of the context effect.

At least two factors may mitigate the degree to which increased familiarity and feature set size translate into increased feature measures. One is the relative proximity of the stimuli in a set. A second is the categorical level of the stimuli. The theoretical arguments and specific hypotheses for these mitigating factors are detailed in the following sections.

\subsection{Relative proximity}

We predict that stimuli at either extreme of relative proximity in a set are relatively unaffected by whether a similarity or dissimilarity scale is used. Only at more intermediate levels may the similarity or dissimilarity nature of the scale have much effect. This prediction is based on the presumption that the variance in feature measures or salience driving Tversky's prediction is minimal at the extremes and maximized among moderately proximal stimuli.

For most consumer product categories there are typically a small number of salient aspects on which consumers group and distinguish stimuli across the category (e.g., the cola versus non-cola and diet versus non-diet nature of soft drinks). Using a memory probe, Johnson (1986) found that consumers only elicited an average of five associated features when prompted with individual soft drinks, beers, and fruits. Information processing studies likewise suggest that consumers seldom use more than four or five attributes when evaluating choice alternatives (Jacoby et al., 1977; Lussier and Olshavsky, 1979; Brucks, 1985). That product attributes are typically skewed in their importance weights, where only a relatively small number of attributes have substantive impact on judgment and choice, is consistent with the growing number of studies that support hierarchical or elimination-type choice models (Hauser, 1986; Johnson, 1988; Johnson and Payne, 1985; Klein and Bither, 1987; Tversky and Sattath, 1979). If 
consumers rely primarily on a small number of important attributes, it follows that there are also a number of less important or secondary attributes that consumers may or may not use (e.g., manufacturer, packaging, etc.). The influence of these secondary attributes should increase with product familiarity and the resulting store of product related attributes in memory (Alba and Hutchinson, 1987; Howard, 1977).

It is reasonable to expect that the more important attributes, whether they be common or distinctive, affect similarity and dissimilarity judgments alike. In contrast, the more secondary attributes should have their greatest influence at an intermediate level of relative proximity. Consider that relatively similar or dissimilar products in a set likely have all or most of the more perceptually diagnostic attributes or aspects as either common or distinctive features. No matter what other attributes are common or unique to particular stimuli, these products will be judged as either very similar (not at all dissimilar) or very dissimilar (not at all similar). The relative influence of the secondary attributes should be minimal. For stimuli between the extremes there is no overriding combination of important common or distinctive features which limits the variability in their feature measures. This should increase the likelihood of a similarity/dissimilarity context effect.

Thus there may be perceptual floor and ceiling effects within any given stimulus set which mitigate any context effect at the extremes. Consider that any two diet colas (e.g., Diet Pepsi and Diet Coke) may be so similar compared to the other soft drinks that they will always be rated as very similar (not at all dissimilar). There are two salient common features that result in a high level of perceived similarity no matter how relatively familiar the soft drinks are. At the other extreme, there are pairs of soft drinks which differ in both their diet versus non-diet and cola versus non-cola distinctions (e.g., Diet Coke and Seven Up, or Diet Sprite and Pepsi). Even as consumers become more familiar with these products and have a greater store of associated features, there are two salient distinctive features that should again result in relatively equivalent feature measures. Now consider more moderately proximal 
pairs in the set (e.g., Diet Coke and Pepsi, or Diet Coke and Diet Seven Up). Intuitively, the more secondary features of these stimuli should play a much greater role in determining their perceived proximity. Here, the more familiar the pair of soft drinks for any given consumer, the higher its similarity plus dissimilarity rating. This suggests a second hypothesis as an alternative to the general context effect predicted under hypothesis one. Hypothesis two posits an interaction between a similarity/dissimilarity context manipulation and the relative proximity of the stimuli in a set.

\section{$\mathrm{H} 2$ : A similarity/dissimilarity context effect is more likely at an intermediate level of relative proximity} than at either extreme.

\subsection{Category level}

A second factor that should mitigate the potential for a similarity/dissimilarity context effect is the categorical level of the stimuli involved. Johnson et al. (1990a) report that subject fatigue has a greater effect on perceptions among brands from the same category than among products that cross category boundaries. As Johnson et al. (1990b) argue, these results are likely due to the salient category differences that more consistently affect judgments involving product category level stimuli. This suggests that category level stimuli (e.g., beverages) should have feature measures that are relatively insensitive to differences in familiarity and associated features. More homogeneous brand level stimuli

(e.g., soft drinks) should have feature measures that are more sensitive to differences in familiarity, which should increase the likelihood of a similarity/dissimilarity context effect.

Our prediction regarding brands versus categories rests on the notion that there is a particularly salient or "basic" level of categorization at which categories are most differentiated from one another (Rosch et al., 1976). That is, they exhibit a singularly high degree of inclusiveness or similarity of their 
members (Murphy, 1982; Murphy and Smith, 1982; Rosch, 1975; Rosch et al., 1976). Brand level stimuli are more likely to belong to the same basic level category while category level stimuli are more likely to cross basic level categories. As defined here, category level stimuli contain $n$ stimuli from $k$ basic level categories where $\mathrm{k}<\mathrm{n}$ and $\mathrm{k}>1$. One example is beverage stimuli that include diet and non-diet soft drinks, fruit juice and lemonade, and milk and chocolate milk. The basic level distinctions should dominate the feature measures of these category level stimuli. Membership in the same or a different basic level category should drive the judgments, be they similarity judgments or dissimilarity judgments. The marginally important within-category similarities and differences that vary in salience with stimulus familiarity should have relatively little effect.

The discussion suggests another interaction-based hypothesis, where a similarity/dissimilarity context effect depends on the categorical level of the stimuli involved, which we state as hypothesis three.

H3: A similarity/dissimilarity context effect is more likely among brand level stimuli than among category level stimuli.

We now report on the empirical study designed to test these hypotheses.

\subsection{Study one: Method and procedure}

Our dependent variable of interest is $[s(a, b)+d(a, b)]$. The main independent variables in the study are the relative familiarity of the stimulus pairs, the categorical level of the stimulus set (brand versus category), and the relative proximity of the pairs within each stimulus set. Independent similarity and dissimilarity judgments were collected for each of four different stimulus sets using a between- 
subjects design. In the similarity task, subjects rated the products on a seven-point scale ranging from 1 (not at all similar), to 7 (very similar). In the dissimilarity task, subjects rated the products on a sevenpoint scale ranging from 1 (not at all dissimilar), to (7 very dissimilar). Following Tversky and Gati (1978), each pair of products was embedded in a separate sentence ("How similar (dissimilar) are product $\mathrm{X}$ and product $\mathrm{Y}$ ?") to maximize the potential for a similarity/dissimilarity context effect. The pairs were rated in one random order by approximately half of the subjects in each condition and in reverse order by the other half.

The stimuli included four of five sets used in the Johnson et al. (1990a) fatigue study, two brand level sets and two category level sets. These stimuli were selected because they meet the conditions of brand and category level stimuli described earlier. Each subject provided paired comparison proximity ratings for one of the four sets. Two sets (beverages and snacks) include stimuli that cross category boundaries while two sets (soft drinks and candy bars) include brands from the same category. Each stimulus set involved 12 products for a total of 66 paired comparison judgments per subject.

The relative proximity of the pairs was operationalized as follows. For each stimulus set we obtained an average similarity and average dissimilarity for each product pair. We converted the dissimilarity averages to a 1 to 7 similarity scale (i.e., $8-d(a, b))$ and then averaged the two measures to obtain an overall average proximity, $p(a, b)$, for each pair across all the subjects, where $p(a, b)=(s(a, b)+$ $(8-d(a, b))) / 2$. The pairs in each set were categorized using this measure into three relative proximity levels, most proximal pairs (where $p(a, b)>5$ ), moderately proximal pairs (where $3<p(a, b) \leqslant 5$ ), and least proximal pairs (where $p(a, b) \leqslant 3$ ). Note that, given the nature of Tversky's proposed context effect, this $p(a, b)$ measure should be unrelated to our dependent variable, $[s(a, b)+d(a, b)]$. Any context effect that increases similarity plus dissimilarity, our dependent variable, should roughly cancel out in the $p(a, b)$ used to operationalize relative proximity. In support of this assumption, the correlation between the $[s(a, b)+d(a, b)]$ and $p(a, b)$ measures was not significant $(r=-0.09, n=264)$. 
Although our three-level categorization of relative proximity is conceptually appropriate given the arguments underlying hypothesis two, one problem is that sample size varies across conditions. Overall, there are only 28 product pairs that fall in the most proximal range versus 69 and 167 in the moderately proximal and least proximal ranges. Thus the average number of most proximal pairs in each stimulus set is only 7 . One solution is to create equally sized relative proximity conditions for each stimulus set (i.e., 22 pairs in each condition for each set). This is problematic in that it forces one to include relatively dissimilar pairs in the moderately proximal range and moderately proximal pairs in the most proximal range. While we focus on the categorization based on meaningful scale ranges, we also report results obtained using the equally sized relative proximity conditions as a check.

Finally, a measure of the subjects' experience with the products was used to operationalize the relative familiarity of the pairs. After the proximity rating task, each subject was asked to rate his or her frequency of consumption for each of the twelve products in the set on a five point scale (every day $=1$, every week $=2$, every month $=3$, every year $=4$, and never $=5$ ). This measure was chosen because familiar products are those with which consumers have the most direct interactions and resulting product knowledge (Alba and Hutchinson, 1987). Empirically, other data also suggested that a frequency of consumption measure closely reflects differences in associated features as assumed. 2 The contrast model predicts a similarity/dissimilarity context effect when pairs of stimuli have both more common and more distinctive features than other pairs. Pair familiarity is operationalized as the product of the pairwise consumption measures to properly capture this precondition of joint familiarity.

Subjects were recruited from graduate and undergraduate business courses and paid for their participation. The study was run in groups of approximately 40 using a questionnaire format. A total of 156 subjects participated, 38, 39, 40, and 39 subjects respectively for the beverages, snacks, soft drinks, and candy bars. The subjects were equally split between the similarity and dissimilarity conditions (78 each). 


\subsection{Study one: Analysis and results}

Initial regression analyses reveal a high level of consistency between the independent similarity and dissimilarity judgments. These judgments were highly correlated $(r=-0.98,-0.93,-0.97$, and -0.96 respectively for the soft drinks, candy bars, beverages, and snacks). This suggests that a similarity/dissimilarity context effect is either highly localized or absent.

An overall analysis of variance was used to provide an initial test of the hypotheses in which each rated pair of products serves as an observation ( $n=264 ; 66$ pairs for each of four stimulus sets). It was expected that average consumption experience increases significantly from brands to categories (Johnson et al., 1992). We standardized our experience-based familiarity measure within each stimulus set to control for such differences. The dependent variable is $[s(a, b)+d(a, b)]$, while the independent variables included our continuous measure of pair familiarity, a three-level relative proximity factor (most, moderately, and least proximal pairs), a two-level category level factor (brands and categories), and two nested variables to capture differences between the two qualitatively different stimulus sets at the brand and category levels. Also included were the two- and three-way interactions involving pair familiarity, category level, and relative proximity.

The overall ANOVA shows no main effect for pair familiarity, relative proximity, or stimulus level on $[s(a, b)+d(a, b)]$. Thus hypothesis one, which posits a general similarity/dissimilarity context effect, was not supported. The only significant two-way interaction $(F(2,250)=3.79, p<0.05)$ was that involving relative proximity and stimulus level on $[s(a, b)+d(a, b)]$. There was, however, a marginally significant three-way interaction involving familiarity, relative proximity, and stimulus level $(F(2,250)=2.52, p$ $<0.10)$. Although not directly relevant to our hypotheses, there were significant nested effects for the two sets of brands $(F(1,250)=17.62, p<0.001)$ and two sets of categories $(F(1,250)=70.25, p<$ 
0.001). The mean for $[s(a, b)+d(a, b)]$ was 7.96 for brands (7.83 for soft drinks, 8.08 for candy bars) versus 7.79 for categories ( 8.03 for beverages, 7.56 for snack foods). As hypotheses two and three make particular predictions regarding the pattern of results across conditions, separate ANOVAs were conducted to more directly test these hypotheses.

To test hypothesis two, separate analyses were conducted for each separate level of relative proximity. For the most proximal pairs, there was no significant increase or decrease in $[s(a, b)+d(a, b)]$ with familiarity. For the moderately proximal pairs, $[s(a, b)+d(a, b)]$ increased significantly with familiarity $(F(1,82)=4.72, p<0.05)$ as predicted by hypothesis two. Although hypothesis three predicts that this main effect is driven by an interaction involving category level, this interaction was not significant. Finally, for the least proximal pairs, there was no significant increase or decrease in $[s(a, b)+$ $d(a, b)]$ with familiarity. This pattern of results supports hypothesis two in that the similarity/dissimilarity context effect only occurs among our moderately proximal stimuli.

To test hypothesis three, separate analyses were conducted for each stimulus level: brands and categories. This hypothesis assumes at least equal or greater variance in familiarity for our categories versus our brands. Otherwise differences in familiarity will necessarily be less determinant of context effects at the category level. The standard deviation in our familiarity variable was slightly greater for the category level stimuli (1.08 versus 0.73 ), which alleviates this concern. In the ANOVAs for brands and categories, the main effect of familiarity on $[s(a, b)+d(a, b)]$ was not significant in either case. However, given the support for hypothesis two, the appropriate test for hypothesis three is the existence of a familiarity by relative proximity interaction for brands but not categories. This interaction was not significant for brands, but marginally significant for categories $(F(2,125)=2.95, p<0.10)$. That is, the support for hypothesis two is concentrated among the category level stimuli rather than the brands. Thus hypothesis three was not supported. 
Recall that, as a check, we also tested the hypotheses using a three-level classification of relative proximity in which the sample sizes were equal across conditions. The results were very consistent with those reported above.

Table 1 presents the correlations between our familiarity measure and the three proximity measures of interest, $[s(a, b)+d(a, b)], s(a, b)$, and $d(a, b)$ to illustrate these relationships. Consistent with hypothesis two, the only condition in which we find a consistent positive relationship between $[s(a, b)+$ $d(a, b)]$ and familiarity across stimulus sets is for the moderately proximal pairs. Consistent with the ANOVA, the correlations are also larger for the category level stimuli than for the brands. Dissimilarity increases and similarity de creases with familiarity across the sets, which is consistent with the argument that product categorization proceeds by a process of grouping and distinguishing. As familiarity grows, consumers learn more about the differences among products in any given category. Finally, the relatively small of most proximal pairs results in more correlations for this condition across the sets.

\section{Study two: Priming task}

Overall the results of study one support the contention that a similarity/dissimilarity context effect is most prevalent among the moderately proximal stimuli in a set. The primary goal of study two is to examine the generalizability of this result to a qualitatively different context effect. A secondary goal is to examine a context effect that is more likely to reveal important differences between brand level and category level stimuli.

A priming task was chosen for several reasons. It is qualitatively quite different from the context manipulation in study one. Previous research has shown that priming tasks, designed to increase the salience of particular attributes or criteria, systematically affect consumers' evaluative judgments and choices (Alba and Hutchinson, 1987; Bettman and Sujan, 1987). The contrast model and hypotheses 
described under study one also apply directly to this type of task. Finally, unlike the similarity/dissimilarity effect, a priming task predicts different directional effects on the perceived proximity of brands and categories making it more likely to reveal any brand versus category differences.

Table 1

Study one: Correlations between pairwise familiarity and proximity measures by relative proximity

\begin{tabular}{|c|c|c|c|c|}
\hline \multirow[t]{2}{*}{ Stimulus set } & \multirow[t]{2}{*}{ Familiarity versus } & \multicolumn{3}{|c|}{ Relative proximity } \\
\hline & & $\begin{array}{l}\text { Most proximal } \\
\text { pairs }\end{array}$ & $\begin{array}{l}\text { Moderately } \\
\text { proximal pairs }\end{array}$ & $\begin{array}{l}\text { Least proximal } \\
\text { pairs }\end{array}$ \\
\hline Soft drinks & $\begin{array}{r}s(a, b)+d(a, b) \\
s(a, b) \\
d(a, b)\end{array}$ & $\begin{array}{c}0.690 \\
(10)^{\mathrm{a}} \\
0.282 \\
0.361\end{array}$ & $\begin{array}{c}0.142 \\
(15) \\
0.392 \\
-0.317\end{array}$ & $\begin{array}{l}-0.296 \\
(41) \\
-0.143 \\
-0.036\end{array}$ \\
\hline Candy bars & $\begin{array}{r}s(a, b)+d(a, b) \\
s(a, b) \\
d(a, b)\end{array}$ & $\begin{array}{l}-0.411 \\
(4) \\
0.080 \\
-0.579\end{array}$ & $\begin{array}{l}0.068 \\
(21) \\
-0.362 \\
0.455\end{array}$ & $\begin{array}{l}0.147 \\
(41) \\
-0.407 \\
0.466\end{array}$ \\
\hline Beverages & $\begin{array}{r}s(a, b)+d(a, b) \\
s(a, b) \\
d(a, b)\end{array}$ & $\begin{array}{l}-0.649 \\
(6) \\
-0.431 \\
-0.347\end{array}$ & $\begin{array}{l}0.476 \\
(18) \\
-0.595 \\
0.702\end{array}$ & $\begin{array}{l}0.089 \\
(42) \\
-0.133 \\
0.166\end{array}$ \\
\hline Snack foods & $\begin{array}{r}s(a, b)+d(a, b) \\
s(a, b) \\
d(a, b)\end{array}$ & $\begin{array}{l}0.292 \\
(8) \\
-0.147 \\
0.386\end{array}$ & $\begin{array}{c}0.311 \\
(15) \\
0.321 \\
-0.223\end{array}$ & $\begin{array}{c}0.004 \\
(43) \\
0.078 \\
-0.056\end{array}$ \\
\hline Overall & $\begin{array}{r}s(a, b)+d(a, b) \\
s(a, b) \\
d(a, b)\end{array}$ & $\begin{array}{c}0.138 \\
(28) \\
-0.039 \\
0.169\end{array}$ & $\begin{array}{l}0.130 \\
(69) \\
-0.024 \\
0.117\end{array}$ & $\begin{array}{l}-0.007 \\
(167) \\
-0.088 \\
0.077\end{array}$ \\
\hline
\end{tabular}

Sample size.

\subsection{Hypotheses}

Priming tasks act as a means of spreading activation within a subject's memory for product information (Anderson, 1983). Within the context of the contrast model, this spread of activation should increase the likelihood that associated product features will affect subsequent similarity ratings. This all assumes that there is a sufficient level of product experience and resulting associated features to be activated by the prime (Murphy and Medin, 1985). The hypotheses that follow are developed with these 
relative "expert" consumers in mind and our initial empirical analysis will focus on validating this assumption.

The directional effect of a priming task should depend on whether brands or categories are being judged. Brands are, by definition, relatively similar alternatives from the same basic-level category which have predominantly common features. A priming task that activates these predominantly common features should, therefore, increase subsequent perceptions of proximity. Advertising research supports a similar hypothesis in that comparative ads increase the perceived similarity of brands (Gorn and Weinberg, 1984; Johnson and Home, 1988). When stimuli cross salient category boundaries there should be a relative predominance of distinctive features. A priming task that activates these predominantly distinctive features should decrease subsequent perceptions of proximity. This prediction also mirrors that developed in an advertising context where comparative ads may differentiate products that cross salient category boundaries (Walker et al., 1986). The discussion suggests two general hypotheses.

H4: A priming task should increase the perceived proximity of brand level stimuli.

H5: A priming task should decrease the perceived proximity of category level stimuli.

However, as in study one, we expect perceptual floor and ceiling effects on product feature measures at the extremes of proximity within a stimulus set. The more secondary product associations of experienced subjects, when primed, should influence those judgments involving moderately proximal stimuli. Unlike relatively similar or dissimilar stimuli in a set, there is no overriding combination of important common or distinctive features that limits the variability in feature measures and subsequent proximity judgments. 
H6: Priming task effects on proximity judgments are more likely at an intermediate level of relative proximity than at either extreme.

Finally, as argued previously, the existence of salient category differences may limit the degree to which a priming manipulation affects categories as opposed to brands.

H7: Priming task effects on proximity judgments are more likely among brand level stimuli than among category level stimuli.

These hypotheses are tested in study two.

\subsection{Study two: Method and procedure}

Two different priming conditions were used in the empirical study, general attribute primes and specific attribute primes. Pre-tests revealed no difference between the two priming conditions, suggesting that each condition primed the same set of product associations. In the analyses that follow, the two types of primes are treated as a single condition with potential nested effects to account for the particular type of prime used. Thus the experimental manipulations included a two-level priming factor (prime versus no prime), a two-level nested factor under the priming condition (specific versus general prime), and a two level factor for brand versus category level stimuli in a between-subjects design.

Subjects were randomly assigned to one of six conditions: (1) category level stimuli/specific primes, (2) categories/general primes, (3) categories/no primes, (4) brand level stimuli/specific primes, (5) brands/general primes, or (6) brands/no primes. The stimuli included the twelve candy bars and twelve 
snack foods from study one. Ninety-six student subjects were recruited for the experiment and paid for their participation.

Each subject received three items: 1) the priming task, 2) the main task, and 3) a single sheet for comments and questions. Subjects were told that they would be participating in two unrelated studies and were instructed to complete Study A (the priming task) before beginning Study B (the main task). After finishing the main task, subjects had the opportunity to write down any questions and/or comments they had about either study. None of the participants questioned the independence of the two studies.

\subsection{Priming task}

An initial questionnaire contained a task to prime the subjects' knowledge of the candy bar or snack food stimuli. As noted, each participant was randomly placed into a general prime condition, a specific prime condition, or into a no prime control condition. The priming manipulation follows Bettman and Sujan's (1987) study in which subjects were primed with a level of processing type task. Bettman and Sujan constructed three word lists of ten words each; each list was relevant to one of the priming conditions. Subjects were first presented with the two word lists not relevant to their condition and asked to perform a structural processing task (determining whether the word contained the letter "e"). The relevant word list was then processed semantically by asking subjects to think of a product described by that word. This priming manipulation ensures that all subjects process all words, the relevant ones at a "deep" level and the nonrelevant words at a "shallow" level.

In our study, three word lists were constructed: a general and a specific list for the prime condition and a neutral list for the no prime condition. The general and specific word lists were generated during a memory probe pretest using two separate samples of 15 subjects drawn from the 
same population as the main part of the study. This was a free-association task in which the subjects were asked to write down the first ten words that came to mind after being prompted with "snack" as a general cue or "chocolate" as a specific cue. Ten attributes, the cue and the nine attributes mentioned most frequently in response to the cue, were used to prime the subjects. These attributes included snack, meal, eat, food, munch, treat, nibble, edibles, nosh, and morsel for the general prime list and chocolate, dark, brown, carob, cocoa, sweet, sugar, milk, mocha, and hazel for the specific prime list. Finally, ten neutral words for the no prime condition were selected from Paivio's list of nouns rated on generality-specificity (Paivio, 1971). These ten words (amount, clean, comedy, health, pressure, month, length, pact, joke, occasion) represent neutral words in that they fall around the midpoint of the specificity-generality scale.

Consistent with our cover story that subjects would be volunteering for two independent studies, the subjects were told they would first be participating in a pre-test to be used later as part of a larger study on word perceptions. They were first presented with the two non-target word lists. Students performed a structural processing task on these twenty words by circling each word that contained the letter "a". After finishing this, they then encountered a target list of the ten words relevant to their priming condition, which they were to process semantically: the general or the specific attribute list for the prime condition and the neutral word list for the no prime condition. The subjects were instructed to read each word and think of a unique sentence using that word. To check that the relevant list was processed at a deeper level than the non-relevant lists, the last page of the questionnaire queried students to recall as many of the 30 words as they could. Results of the manipulation check indicate that the subjects recalled significantly more semantically processed words (7.11) than structurally processed words $(1.92 ; t=15.40, p<0.001)$.

\subsection{Main task}


Immediately after completing the priming study, subjects were told they would be participating in a new study on consumer products. Their first task was to rate the pairwise proximity of different products. Half of the subjects received brand level products consisting of the twelve candy bar brands from study one. The other half received twelve category level products consisting of the snack foods from study one. The subjects were first shown a list of all the stimuli they would be rating and a sample rating scale. Subjects then rated the perceived proximity of each pair of products on a seven point scale ranging from 1 (very similar) to 7 (very dissimilar). Note that, in contrast to experiment 1, our discussion and hypotheses focus on a neutral "very similar to very dissimilar" proximity rating scale. This scale was reverse coded in subsequent analyses so that higher values represent increased proximity. Half of the subjects rated the pairs in random order and the other half in the reverse order for each stimulus set.

The relative proximity and familiarity of the pairs were operationalized in the same fashion as in study one. Because the sample sizes for relative proximity were again small in the most proximal condition ( $n=6$ and 9 for brands and categories), we again checked the relative proximity hypothesis using a relatively proximity variable in which sample sizes were constrained to be equal across conditions.

\subsection{Study two: Analysis and results}

As in study one, initial regression analyses reveal a high level of consistency between the pairwise proximity judgments for the prime and no prime conditions. These judgments were correlated 0.90 and 0.96 for the candy bar pairs $(n=66)$ and snack food pairs $(n=66)$, respectively. As for the similarity/dissimilarity effect, this suggests that the priming effect is again either highly localized or nonexistent. 
Analysis of variance was used to test our assumption that a priming task affects those subjects who are relatively familiar with the products being judged. Our dependent variable here and in subsequent analyses is each subject's average proximity rating for the relevant product pairs. A median split of average familiarity across products was used to categorize the subjects into two levels of relative familiarity for each stimulus set: experts and novices. Both the experts and novices were included in an initial overall ANOVA. This analysis reveals a significant three-way interaction of stimulus level by prime by familiarity on average similarity $(F(1,87)=4.23, p<0.025)$. We then examined the novices separately and, as expected, found no support for hypotheses four through seven.

Subsequent analyses focus on the expert subjects to test hypotheses four through seven. Hypotheses four and five were tested with an ANOVA which contained a two-level priming factor (prime versus no prime), a two-level category level factor (brands and categories), a two level nested variable for the type of prime (general versus specific), and the interaction involving the priming and category level factors. The results reveal a significant prime by level interaction $(F(1,45)--4.06, p<0.025)$. Separate analyses for the brands and categories reveal that the priming task increases proximity for the brands $(F(1,47)=3.41, p<0.05)$ but has no significant effect on the categories $(F<1)$. These results support hypothesis four but not hypothesis five. This pattern of results also supports hypothesis seven, which posits that any priming effects are likely to be stronger for brands than for categories.

Hypothesis six predicts that the effects are not general but localized among the moderately proximal pairs. As in study one, an explicit test of this hypothesis requires an analysis of effects for each separate level of relative proximity and we con ducted separate ANOVAs for this purpose. For the most proximal pairs, there was no significant prime by level interaction. The only result here is that the most proximal categories were more similar than the most proximal brands $(F(1,45)=7.83, p<0.01)$. There was no significant increase in brand level proximity, or decrease in category level proximity, with the use of a prime. There was a significant prime by level interaction for the moderately proximal pairs $(F(1,45)=$ 
$4.92, p<0.05)$. This interaction is driven by a marginally significant increase in brand level similarity $(F(1,47)--2.27, p<0.07)$ as well as a significant decrease in category level similarity $(F(1,47)=2.70, p<$ 0.05) with the use of a prime. Finally there was a significant prime by level interaction for the least proximal pairs $(F(1,45)=3.90, p<0.05)$, although it was not as strong as that observed for the moderately proximal pairs. This interaction is driven by a significant increase in brand level similarity with the use of a prime $(F(1,47)=4.42, p<0.05)$. There was no significant or near-significant priming effect for the least proximal, category level pairs. There was also a significant main effect for level $(F(1,45)=2.88, p<0.05)$ among the least proximal pairs.

The mean proximity levels are provided in Table 2. They illustrate the general increase in perceived brand level proximity with the use of a prime. Although the priming task decreased the proximity for the categories as predicted, this decrease was not significant across all the pairs. These results support hypotheses four and seven but not hypothesis five. Finally, supporting hypothesis six and consistent with the findings in study one, the priming effects are more prevalent among the moderately proximal stimuli. Re-analysis of the data using the relative proximity variable in which sample size was constrained to be equal across conditions showed essentially the same pattern of results; the priming effects were again more prevalent among the moderately proximal stimuli.

These results suggest that priming a subject's product knowledge increases the salience of existing similarities among brands and differences among categories. Consistent with the predictions made using Tversky's contrast model and the results of study one, this context effect has a greater impact when a pair of products is among the moderately proximal pairs in a set. Study two also suggests that priming effects are greater for brands than for categories.

\section{General discussion}


A number of research studies have identified context effects in consumer judgments. Yet prior research

Table 2

Study two: Proximity judgment means and standard deviations across conditions for expert subjects

\begin{tabular}{|c|c|c|c|c|c|}
\hline \multirow[t]{2}{*}{ Stimulus set } & \multirow{2}{*}{$\begin{array}{l}\text { Priming } \\
\text { condition }\end{array}$} & \multirow[t]{2}{*}{ All pairs } & \multicolumn{3}{|c|}{ Relative proximity } \\
\hline & & & $\begin{array}{l}\text { Most proximal } \\
\text { pairs }\end{array}$ & $\begin{array}{l}\text { Moderately } \\
\text { proximal pairs }\end{array}$ & $\begin{array}{l}\text { Least proximal } \\
\text { pairs }\end{array}$ \\
\hline \multirow[t]{2}{*}{$\begin{array}{l}\text { Brands } \\
\text { (candy bars) }\end{array}$} & prime & $\begin{array}{l}3.79^{\mathrm{a}} \\
(0.53)^{\mathrm{b}}\end{array}$ & $\begin{array}{l}5.44 \\
(0.44)\end{array}$ & $\begin{array}{c}3.88 \\
(0.57)\end{array}$ & $\begin{array}{c}3.10 \\
(0.78)\end{array}$ \\
\hline & no prime & $\begin{array}{c}3.31 \\
(0.78)\end{array}$ & $\begin{array}{c}5.36 \\
(0.64)\end{array}$ & $\begin{array}{c}3.43 \\
(0.85)\end{array}$ & $\begin{array}{c}2.47 \\
(0.77)\end{array}$ \\
\hline Sample size & & & 6 & 40 & 20 \\
\hline \multirow[t]{2}{*}{$\begin{array}{l}\text { Categories } \\
\text { (snacks) }\end{array}$} & prime & $\begin{array}{c}3.26 \\
(0.57)\end{array}$ & $\begin{array}{c}5.94 \\
(0.55)\end{array}$ & $\begin{array}{c}3.48 \\
(0.69)\end{array}$ & $\begin{array}{c}2.33 \\
(0.73)\end{array}$ \\
\hline & no prime & $\begin{array}{c}3.51 \\
(0.73)\end{array}$ & $\begin{array}{c}5.74 \\
(0.56)\end{array}$ & $\begin{array}{c}3.98 \\
(0.92)\end{array}$ & $\begin{array}{c}2.53 \\
(0.77)\end{array}$ \\
\hline Sample size & & & 9 & 25 & 32 \\
\hline
\end{tabular}

a Mean.

b Standard deviation.

is unclear as to whether these context effects pervade consumer judgments of product proximity. This study used Tversky's (1977) contrast model of similarity to hypothesize that these context effects should be more pronounced among the moderately proximal stimuli in a set, and more likely to occur for brands than for categories. The two studies reported here provide support for the first prediction and mixed support for the second.

The results of study one reveal that the similarity/dissimilarity context effect popularized by Tversky is concentrated among moderately proximal stimuli. Even here, there is no general increase in both similarity and dissimilarity judgments with stimulus familiarity as reported in earlier studies (e.g., Tversky and Gati, 1978). Rather, our subjects perceived greater dissimilarity (less similarity) among products as familiarity increased. This is consistent with the general view of category formation as a process of grouping and then distinguishing among products. These results demonstrate that a similarity/dissimilarity context effect does not pervade consumer product judgments. Although we expected the effect to be more likely for brands than for categories, no such difference emerged. Similarity and dissimilarity judgments were very consistent for the brands and categories of beverages and snack foods studied here (overall $r=-0.95$ ). 
Our second study examined whether a priming of consumers' product knowledge would increase the perceived proximity of similar brands and decrease the proximity of more dissimilar categories. While providing another test of the relative proximity hypothesis, this type of effect should be more likely to reveal differences between brand and category level stimuli. The most important finding of this study is that, like the similarity/dissimilarity effect, the priming effect was most prevalent among the moderately proximal stimuli in a set. This supports our contention that context effects have a localized effect on a subset of products in a set. The study was also successful in showing that priming effects are more prominent among brands than among categories. This is consistent with Johnson et al.'s (1990a) study of the effects of fatigue on proximity and the notion that category level differences are more salient than brand level differences.

The primary implication of these results concerns the interchangeability of alternative proximity measures. For those worried that subtle context manipulations or priming effects add systematic biases to product judgments, our results are encouraging. This was not the case in either study; task and context effects did not systematically plague our consumers' judgments. Again, the effects that did occur were localized among the moderately proximal products in a category.

This, in turn, provides insight into the debate over whether or not proximity can be used to predict preferences. Marketers often use positioning maps to explain and predict consumers' strength of preference for alternative brands. These maps presume that proximal brands are more substitutable or equally preferred by consumers. This assumption has been challenged by empirical evidence that proximity and preference judgments are often discrepant (Shocker et al., 1990; Elrod, 1988). One explanation for this discrepancy is that proximity judgments are inherently unstable and systematically biased by context and task effects. This argument has been advanced in both psychology and marketing (Malhotra, 1990; Medin et al., 1990). Our results downplay this possibility and suggest that it is only a viable explanation among the more moderately proximal stimuli in a set. An interesting proposition for 
future research would be to test whether the relationship between proximity and preference is stronger among the most proximal and least proximal product pairs in a set than among moderately proximal pairs. Our results also lend credence to an alternative explanation for a preference-proximity discrepancy, as suggested by Lefkoff-Hagius and Mason (1990), which is that the relative importance of attributes varies between proximity and preference judgments.

A second implication relates to how we measure proximity and related constructs in marketing research. Similarity and dissimilarity scales are used to operationalize not only perceived proximity but also prototypicality (Loken and Ward, 1990) and brand equity manipulations (Keller, 1991). Although alternative proximity measures may not be completely interchangeable, they are generally consistent. This suggests that we view perceived proximity as a latent or unobservable variable that is reliably reflected in alternative measures and scales (Fornell, 1989). Thus one could use a similarity judgment, a dissimilarity judgment, and subject-referent judgments (i.e., how similar is a to b?) as multiple indicators of some underlying level of proximity. These proximity judgments would also be useful when operationalizing latent prototypicality. The more proximal, on average, any given stimulus is to the other products in a set, the more prototypical the stimulus is of the set as a whole (Nosofsky, 1988). Reliable multiple measures of prototypicality could thus be obtained from alternative proximity judgments. As an indication of this, we calculated the average proximity of each beverage alternative from study one to the other eleven beverages in the set using both the similarity and dissimilarity judgments. The resulting prototypicality measures were correlated 0.94 .

That we should view proximity as a latent variable is consistent with recent developments in the scaling literature. DeSarbo et al.'s (1992) TSCALE procedure rests on this presumption. These authors argue that whether products are cognitively represented using dichotomous features or continuous dimensions, their properties may be captured at a more abstract level using latent dimensions. Their procedure uses alternative proximity measures to operationalize Tversky's contrast 
model at a latent variable level. DeSarbo et al. illustrate the procedure using both fast food restaurants and soft drinks as stimuli. An interesting result in their study parallels ourfindings. To operationalize latent proximity, subjects were asked to judge directionally how similar product a was to product b as well as how similar product b was to product a. Tversky (1977) reports that, when one stimulus has more distinctive features than another, this subject-referent context manipulation should result in systematically different judgments. However, these judgments were minimally different and provided good multiple measures of latent proximity.

A possible limitation of our studies is that the stimuli may have been limited in the degree to which their feature measures could vary. This may have kept any observed context effects to a minimum. For example, our soft drink and candy bar stimuli included only national brands. Using national as well as local brands may have increased the familiarity and knowledge differences and the potential for context effects. However, even with this limitation, we found a predictable context effect among our moderately proximal stimuli in both studies as well as a general priming effect for our brands in study two. Another limitation is that our studies are limited to food and beverage products. The context effects studied here may be more or less prominent among other product types. 


\section{References}

1. Aaker, D.A. and K.L. Keller, 1990. Consumer evaluations of brand extensions. Journal of Marketing 54, 27-41.

2. Alba, J.W. and J.W. Hutchinson, 1987. Dimensions of consumer expertise. Journal of Consumer Research 13, 411-454.

3. Anderson, J.R., 1983. The architecture of cognition. Cambridge, MA: Harvard University Press.

4. Barsalou, L.W., 1983. Ad hoc categories. Memory and Cognition 11, 211-227.

5. Barsalou, L.W., 1985. Ideals, central tendency, and frequency of instantiation as determinants of graded structure in categories. Journal of Experimental Psychology: Learning, Memory and Cognition 11, 629-649.

6. Bettman, J.R. and M. Sujan, 1987. Effects of framing on evaluation of comparable and noncomparable alternatives by expert and novice consumers. Journal of Consumer Research 14, 141-154.

7. Brucks, M., 1985. The effects of product class knowledge on search behavior. Journal of Consumer Research 12, 1-16.

8. Cooper, L.G., 1983. A review of multidimensional scaling in marketing research. Applied Psychological Measurement 7, 427-450.

9. DeSarbo, W.S., M.D. Johnson, A.K. Manrai, L.A. Manrai and E.A. Edwards, 1992. TSCALE: A new multidimensional scaling procedure based on Tversky's contrast model. Psychometrika 57, 4369.

10. Elrod, T., 1988. Choice map: Inferring a product-market map from panel data. Marketing Science 7, 21-40. 
11. Fornell, C., 1989. The blending of theoretical and empirical knowledge in structural equations with unobservables. In: H. Wold (ed.), Theoretical empiricism: A general rationale for scientific model-building, 153-174. New York: Paragon House.

12. Gati, I. and A. Tversky, 1984. Weighting common and distinctive features in perceptual and conceptual judgments. Cognitive Psychology 16, 341-370.

13. Glazer, R., B.E. Kahn and W.L. Moore, 1991. The influence of external constraints on brand choice: The lone-alternative effect. Journal of Consumer Research 18, 119-127.

14. Gorn, G.J. and C.B. Weinberg, 1984. The impact of comparative advertising on perception and attitude: Some positive findings. Journal of Consumer Research 11, 719-727.

15. Hauser, J.R., 1986. Agendas and consumer choice. Journal of Marketing Research 23, 199-212.

16. Howard, J., 1977. Consumer behavior: Application of theory. New York: McGraw-Hill.

17. Jacoby, J., G.J. Szybillo and J. Busato-Schach, 1977. Information acquisition behavior in brand choice situations. Journal of Consumer Research 3, 209-216.

18. Johnson, E.J. and J.W. Payne, 1985. Effort and accuracy in choice. Management Science 31,395414.

19. Johnson, M.D., 1981. Context effects in product perception. In: K.B. Monroe (ed.), Advances in consumer research, Vol. 8, 112-115. Ann Arbor, MI: Association for Consumer Research.

20. Johnson, M.D., 1986. Consumer similarity judgments: A test of the contrast model. Psychology \& Marketing 3, 47-60.

21. Johnson, M.D., 1988. Comparability and hierarchical processing in multialternative choice. Journal of Consumer Research 15, 303-314.

22. Johnson, M.D. and D.A. Home, 1988. The contrast model of similarity and comparative advertising. Psychology \& Marketing 5, 211-232. 
23. Johnson, M.D., D.R. Lehmann, C. Fornell and D.R. Home, 1992. Attribute abstraction, featuredimensionality, and the scaling of product similarities. International Journal of Research in Marketing 9, 131-147.

24. Johnson, M.D., D.R. Lehmann and D.R. Home, 1990a. The effects of fatigue on judgments of inter product similarity. International Journal of Research in Marketing 7, 35-43.

25. Johnson, M.D., D.R. Lehmann and D.R. Home, 1990b. Alternative explanations for changes in similarity judgments and MDS structure. International Journal of Research in Marketing 7, 53-56.

26. Keller, K.L., 1991. Conceptualizing, measuring, and managing customer-based brand equity. Journal of Marketing 57, 1-22.

27. Klein, N.M. and S.W. Bither, 1987. An investigation of utility-directed cutoff selection. Journal of Research 14, 240-256.

28. Lefkoff-Hagius, R. and C.H. Mason, 1990. The role of tangible and intangible attributes in similarity and preference judgments. In: M.E. Goldberg et al. (eds.), Advances in consumer research, Vol. 17, 135-143. Provo, UT: Association for Consumer Research.

29. Loken, B. and J. Ward, 1990. Alternative approaches to understanding the determinants of typicality. Journal of Consumer Research 17, 111-126.

30. Lussier, Denis and Richard Olshavsky, 1979. Task complexity and contingent processing in brand choice. Journal of Consumer Research 6, 154-165.

31. Malhotra, N.K., 1990. Comments on 'The effects of fatigue on judgments of interproduct similarity'. International Journal of Research in Marketing 7, 45-51.

32. Medin, D.L., R.L. Goldstone and D. Gentner, 1990. Similarity involving attributes and relations: Judgments of similarity and difference are not inverses. Psychological Science 1, 64-69.

33. Murphy, G.L., 1982. Cue validity and levels of categorization. Psychological Bulletin 91, 174-177. 
34. Murphy, G.L. and D.L. Medin, 1985. The role of theories in conceptual coherence. Psychological Review 92, 289-316.

35. Murphy, G.L. and E.E. Smith, 1982. Basic-level superiority in picture categorization. Journal of Verbal Learning and Verbal Behavior 21, 1-20.

36. Nosofsky, R.M., 1988. Similarity, frequency, and category representations. Journal of Experimental Psychology: Learning, Memory, and Cognition 14, 54-65.

37. Paivio, A., 1971. Imagery and verbal processes. New York: Holt, Rinehart and Winston.

38. Rosch, E., 1975. Cognitive representation of semantic categories. Journal of Experimental Psychology: General 104, 192-233.

39. Rosch, E., 1977. Human categorization. In: N. Warren (ed.), Studies in cross-cultural psychology: Volume I, 1-49. London: Academic Press.

40. Rosch, E., C.B. Mervis, W.D. Gray, D.M. Johnson and P. Boyes-Braem, 1976. Basic objects in natural categories. Cognitive Psychology 8, 382-439.

41. Shocker, A.D. and V. Srinivasan, 1979. Multi-attribute applications for product concept evaluation and generation: A critical review. Journal of Marketing Research 15, 159-180.

42. Shocker, A.D., D.W. Stewart and A.J. Zahorik, 1990. Determining the competitive structure of product-markets. Working paper, "Carlson School of Management. Minneapolis, MN: University of Minnesota.

43. Tversky, A., 1977. Features of similarity. Psychological Review 84, 327-352.

44. Tversky, A. and I. Gati, 1978. Studies of similarity. In: E. Rosch and B. Lloyd (eds.), Cognition and categorization, 79-98. New York: Wiley.

45. Tversky, A. and I. Gati, 1982. Similarity, separability, and the triangle inequality. Psychological Review 89, 123-154.

46. Tversky, A. and S. Sattath, 1979. Preference trees. Psychological Review 86, 542-573. 
47. Walker, B.A., J.L. Swasy and A. Rethans, 1986. The impact of comparative advertising on perception formation in new product introductions. In: R.J. Lutz (ed.), Advances in consumer research, Vol. 13, 121-125. Provo, UT: Association for Consumer Research. 